\title{
Analysis on the Application of the Over-sampling Technology in the Communication Signal Processing
}

\author{
WANG Juan ${ }^{1, a}$ \\ ${ }^{1 .}$ Chongqing College Of Electronic Engineering, Chongqing 401331,China \\ awj8888886@126.com
}

Keywords: Communication; Signal processing; Over-sampling technology

\begin{abstract}
When dealing with communication signals before the signal transmission, the modulation signal is given priority to the periodic signal, so the modulated signal already have the characteristics of the cyclical before sending actually. In order to simplify the process, the signal hypothesis is generally for stationary signal, however in practice, the communication signal is already with stability and circularity. Through over-sampling, it can make the received signal to be cycle stability, and can reflect the change of signal statistics in time, making up for the deficiency of the stationary signal on the processing method. This article will focus on the application of the over-sampling technology in the communication signal processing.
\end{abstract}

\section{Introduction}

Before communication signal processing, the signal transmission through modulation and the modulation signal is usually a periodic signal, so after modulation signal before sending actual has periodic changes. Generally, in order to simplify the processing method assumes that the signal is stable, but in practice, communication signals have smooth circulation characteristics. Cycle stationary signal is between stationary and non-stationary signal processing of a special type of signal, its statistical measuring tool is cyclical, so compared with the non-stationary signal processing method is simpler, and easier to implement. Over-sampling technology is the key technology of communication signals to realize cycle stability, through sampling, the received signal with the cycle stability, can reflect the statistic of the non-stationary signal, make up for the inadequacy of stationary signal processing party and. It is a sampling technique is widely used in signal processing.

\section{The Theory of the Over-sampling Technology}

The so-called over-sampling is refers to receive signals to sampling at a rate higher than that of baud rate. It with higher than the Nyquist rate of the input signal sampling, in certain cases, the total power signal sampling quantization theory is known. If the minimum input signal amplitude is bigger than the quantize quantization steps, and the input signal amplitude distribution random, so no matter how change, the sampling frequency that the total power of the quantization noise is unchanged, is a constant. Helping signal sampling technology is the key to keep circulation smooth conveying, after sampling, the received signal would be cyclical and stability, not only can reflect the change of the signal statistics in time, and in a timely manner to make up for in dealing with a stationary signal deficiency, therefore, over-sampling technology widely used in signal processing.

Sampling technology is digital signal processing (DSP) is used to improve the AD converter (ADC) performance often use one of the ways it by reducing quantization noise, improve signal-to-noise ratio of the $\mathrm{ADC}$, so as to improve the effective resolution of the ADC. Over-sampling technology not only didn't increase additional analog circuits, and due to increase the effective resolution also simplifies analog circuits, and simple, which are digital signal processing (DSP) practitioners are widely used in measurement and control field. And sampling technology in communication signal processing, over-sampling technology mainly refers to the received signal sampling in higher than the baud rate. With minimal when the input signals 
amplitude bigger than the quantize quantization steps, the input signal amplitude distribution is random. Say simply, it is no matter how sampling frequency would change, the total power of the quantization noise is also needed for will not change, is usually just a constant. Want to ensure that the communication signal can be transmitted smooth continuous cycle; the key is to ensure that the application of the over-sampling technology, by right application of sampling technology can effectively guarantee the received signal to have certain cycle and stable performance. This not only ensure the signal statistics reflect in time, at the same time, also to make up for the signal stability has some effect. So, in the process of handling of communication signals, a sampling technique has been effectively used in it.

\section{The Cycle Smooth Performance of Sampling Signal}

If $x(k)$ is the launch of the limited character sequence for the digital communication system, symbol interval $T$, and $x(k)$ is As independent identically distributed and the mean is zero, namely $E\left[x(k) x^{*}(l)\right]=\sigma_{x}^{2} \delta(l-k) ; h(t)$ is as the impulse response of linear time invariant channel; Under the condition of without considering the noise, received signal is:

$$
y(t)=\sum_{k=-\infty}^{\infty} x(k) h(t-k T)
$$

The receiving signal is over-sampling, sampling interval for $\Delta t=T / P$, As an integer, then the output signal after sampling as follows:

$$
y(n \Delta t)=\sum_{k=-\infty}^{\infty} x(k) h(n \Delta t-k T)
$$

Or write to: $y(n)=\sum_{k=-\infty}^{\infty} x(k) h(n-k T)$

Therefore, after a sampling signals $y(n)$ is cycle stationary signal, and the cycle is $T$.

\section{The Effective Application of the Communication Signal Processing in the Sampling Technology}

(1)The blind equalization and blind identification system

In the traditional communication system, the traditional method of customer service to be interference is achieved by prior knowledge of channel or channels to distinguish and balanced. However, under normal circumstances, access to training sequence is not too realistic, sometimes cost is higher, so people often used method is to blind equalization and blind identification. With the continuous development of society, modern people for blind equalization and blind identification method in the second stage circulation statistics on the use of relatively concentrated, and that the main reason is because of cycle stationary signal processing way than of cycle stationary signal processing method to be able to better resist the noise interference and inhibition. Cycle stationary signal, in general the amount of accumulated by two cycle can be effective for non-minimum phase system identification, to improve the convergence speed while also effectively reduces the amount of computer.

(2)The modulation signal recognition and classification

Usually, for the communication signal modulation signal recognition and classification, its main refers to accept according to the signal, in order to make sure the delivery way of modulation signal, and on this basis, the parameters of modulation, provide important basis for the analysis of the next communication signals. Because cyclic cumulates varies with modulation mode of communication signals, and so, we need to do is to accept the signal sampling in the first place, followed by power spectrum of the signal for further analysis, finally, the cyclic frequency signal carrier frequency and finally determined, it is also a means of communication signal modulation and the premise of the modulation parameters are finalized. 

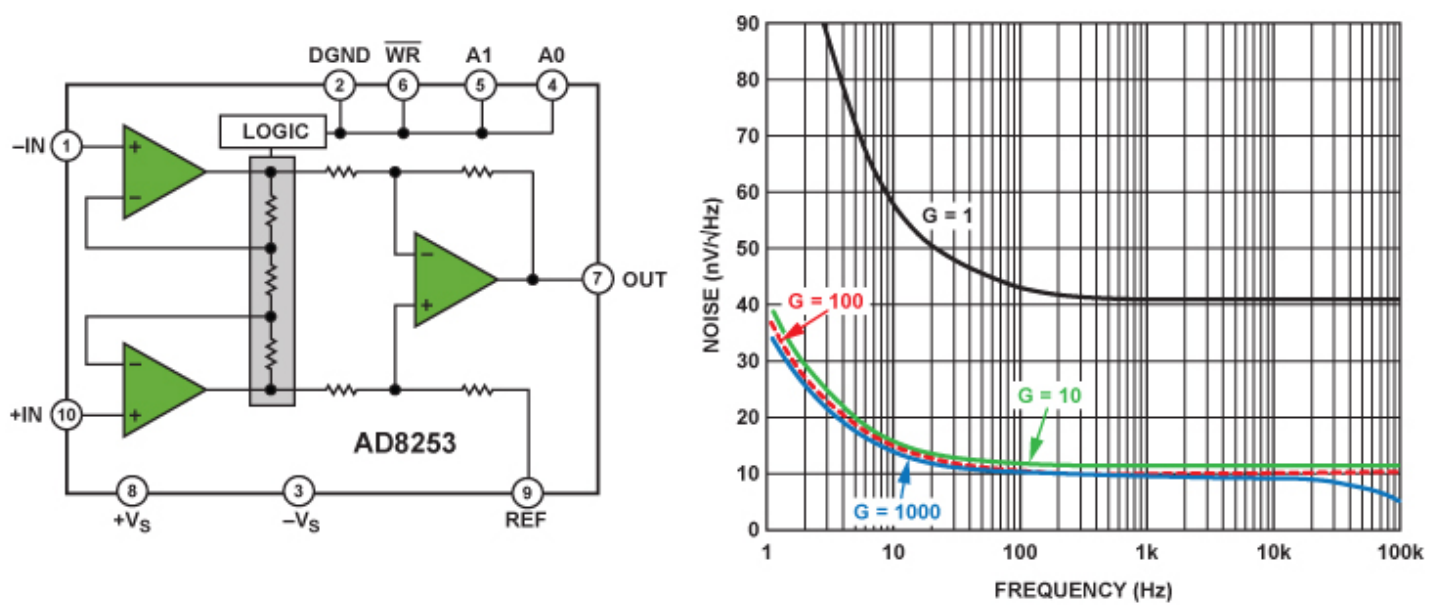

Figure 1 sampling system

(3)The detection signal

Traditional energy detection method in the process of actual application of usually exist some shortcomings, namely when the communication environment is relatively poor, usually for a weak signal with a strong background noise signal detection, the traditional methods cannot effectively detect, moreover background noise to the signal detection by the flood. So, in the modern society, by right application of sampling signal detection method, will guarantee the effective processing on signal characteristics, and through the characteristics of the handling of the sampling signals, to ensure the smooth circulation of information of noise can be zero, at the same time also can ensure to minimize the effect of noise. Therefore, when in complex communication environment, can also be an effective detection of the signal, and can ensure the test results.

\section{Over-sampling rate applications}

The multiple sampling rate technology in modern communication has the wide application of various forms: such as A/D conversion, digital communications, audio and video signal processing and radar signal processing, etc.

(1)The application of the A/D

The sampling rate processing technology is widely used in high-speed ADC. $\Sigma-\Delta$ ADC of high speed signal sampling, expressed in the difference in value between adjacent samples values. High sampling extends the signal to noise to wide frequency band, via multiple sampling filter reduce the sampling rate and the out-of-band noise filtering and then the signal-to-noise ratio can be improved. Time mixed type ADC with multiple low-speed ADC parallel signals such as alternating between sampling to realize high-speed analog-to-digital conversion. The time is not accurate matching of ADC will affect the precision of sampling. Before the A/D with multi-channel QMF signals can be divided into several sub-band, sampling error of each sub-band noninterference, comprehensive and sampling in the digital domain signal eliminate mismatch between the wavelet coefficients. Multiple sampling rate signal processing can make up for a lack of existing ADC performance.

(2)The application of digital communication

Multiple sampling rate signal processing is widely used in the communication system: interpolation filter, timing, synchronization, etc. Before the $\mathrm{D} / \mathrm{A}$ need to increase the sampling rate signal; Demodulation of the ADC to high-speed analog signal sampling, the over-sampling ratio is very big, need to reduce the sampling rate to save overhead. Modern timing recovery in the digital domain, calculate the matched filtering data timing error control after re-sampling filter to calculate the best sampling points, the high precision, simple implementation, has been widely used in modern digital receiver. Decomposition/synthesis filter bank can realize TDMA, CDMA and 
FDMA. Orthogonal frequency division multiplexing (OFDM), discrete multi-tone (DMT) modulation are available such as M channel filter bank.

(3)The application of the audio and video

Filter group theory is used for audio and video compression, coding and recognition. After QMF originally is used for voice compression, and gradually used in image and video compression. Multi-channel filter pair group according to the amount of information coding, abandon sub-band without information of information compression. Through interpolation and superposition of each sub-band can be undistorted signal reconstruction. Multidimensional sampling rate signal processing can be more direct image, video and other multidimensional signal processing.

(4) The application of the radar signal processing

Modern radar receiver for more and more wide input broadband, more and more high sensitivity and resolution, the digital information processing technology and the signal processing ability, by the high quality of radar signal reception task. In radar signal environment, the receiver of the signal frequency band, clutter or some other interference signal frequency also received by receiver. So the radar receiver in order to detect the tiny signal, keep low false alarm probability, which requires the receiver must have a relatively large dynamic range, to keep normal work. Using sampling technology transform radar signal at the receiving end of cycle stationary signal, in addition, after dealing with the sample, the receiver to receive the amount of information will increase, so from the radar signal can obtain more valuable information. In addition, the cycle stationary signal processing and radar signal inhibition of multiplicative noise and additive noise, so after sampling technique to collect more practical radar signal.

\section{Summary}

In a word, with the continuous development of modern communications technology in our country, our country has its own research cycle in view of the signal and smoothness of more and more get the attention of the researchers, and the results also showed that when the features of stable signal can reach the effect of algorithm to simplify and improve the noise ratio, and can realize the value of will be very high. Therefore, in the process of modern signal processing, the cycle stationary signal has become a modern the most focus of attention. Among them, the over-sampling technology as key of cycle stationary signal processing, it will receive more attention, and get more long-term development.

\section{References}

[1] Samueli H.An improved search algorithm for the design of multiplierless FIR filters with powers-of-two coefficients. IEEE Transactions on Circuits and Systems. 2010

[2] Candy, J.,Benjamin, O.The Structure of Quantization Noise from Sigma-Delta Modulation. Communications, IEEE Transactions on. 2011

[3] Candy, J.,Wooley, B.,Benjamin, O.A Voiceband Codec with Digital Filtering. Communications, IEEE Transactions on . 2012

[4] Candy,James C.USE OF LIMIT CYCLE OSCILLATIONS TO OBTAIN ROBUST ANALOG-TO-DIGITAL CONVERTERS. IEEE Transactions on Communications. 2013

[5] Inose, H., Yasuda, Y.,Murakami, J.A Telemetering System by Code Modulation - $\Delta$ इModulation. Space Electronics and Telemetry, IRE Transactions on. 2012

[6] Kazimierz Wiatr,Ernest Jamro.Implementation Image Data Convolutions Operations in FPGA Reconfigurable Structures for Real-Time Vision Systems. Proc.Information Technology, Coding and Computing. 2011 